\title{
MACHO Messages from the Big Bang
}

\author{
George Chapline, ', James Barbieri, ${ }^{2}$ \\ ${ }^{1}$ Lawrence Livermore National Laboratory \\ P.O. Box 808 \\ Livermore, CA 94550, USA \\ ${ }^{2}$ Naval Air Warfare Center - retired \\ 1 Administration Circle \\ China Lake, CA 93555, USA \\ Received: 6 April 2018, Accepted: 10 May 2018, Published: 12 May 2018
}

\begin{abstract}
The present day mass spectrum for dark matter compact objects is calculated based on the assumption that a uniform population of $\mathrm{PBHs}$ was created at a definite redshift with a uniform mass and that the mass spectrum evolved as a result of gravitational radiation. The predicted present day spectrum extends over many decades of mass and allows one to connect the abundance of MACHOs in the halo of our galaxy with the abundance of galactic seeds. Present day astrophysical constraints on the abundance of dark matter PBHs appear to be consistent with our predicted mass spectrum if it is assumed that the seeds for the present day dark matter MACHOs were created at a time $\sim 10^{-4}$ seconds after the big bang. Remarkably the total cosmological energy density at this time obtained by extrapolating the sum of the present day dark matter and CMB energies backward in time and is very close to the mass-energy density in an Einstein- de Sitter universe at the same time. This suggests that the radiation precursor to the CMB was created at about the same time as the seeds for the present day dark matter.
\end{abstract}

\section{DOI: 10.31526/LHEP.1.2018.04}

Some time ago it was suggested [1],[2] that the dark matter component of the matter in today's universe might consist entirely of primordial black holes (PBHs). It was also pointed out [3] in a flat universe whose mass-energy density is dominated by primordial black holes it would perhaps be natural for all matter to eventually consist of horizon mass black holes. Of course these proposals begged the question as to why dark matter should consist of black holes rather than some other exotic form of non-baryonic matter such as WIMPs or axions. On the other hand the persistent failure to find any evidence for WIMPs or cosmic axions has perhaps tipped the balance in favor of PBHs [4],[5] In this letter we focus on the question of what the present day spectrum of dark matter compact objects might have to say about the form of matter near the onset of the big bang. We show that if an initially uniform population of PBHs evolves as a result of gravitational radiation during binary collisions, then the present day mass spectrum for primordial compact objects will smoothly interpolate between the MACHO objects which could form the halo of our galaxy [6] and the massive seeds for the compact objects at the center of galaxies $[7],[8],[9]$. This puts constraints on the redshift where the initial $\mathrm{PBH}$ were formed, which naturally leads to the question as to the form matter took prior to this redshift. An intriguing possibility is that the $\mathrm{PBH}$ precursors to the present day MACHOs and the radiation precursors to CMB were both created at the same redshift by the release of entropy from massive compact objects created at the onset of the big bang.

Our basic assumption is that the present day population of dark matter MACHOs evolved from an initial population of compact objects with nearly the same mass $M_{D M}$ created

\footnotetext{
Email address: barbierijf@hughes . net (James Barbieri, ${ }^{2}$ )

${ }^{1}$ (C) 2018 The Author(s). Published by LHEP.This is an open access article under the CC BY licensehttp://creativecommons .org/licenses/by/4.0/
}

at a specific redshift $z_{r}$.We will also assume that during the radiation dominated era $z<z_{r}$ the evolution of cosmological parameters for the universe proceeds as in the standard cosmological model [10]. In addition we assume that the time corresponding to the redshift $z_{r}$ occurs before the time of cosmological nucleosynthesis, since the creation of precursors to present day dark matter might be expected to affect the standard model nucleosynthesis predictions which are in excellent agreement with observations. With these assumptions we have used Boltzmann-like equation to numerically calculate the dark matter MACHO mass spectrum that would evolve during the radiation era as a result of binary collisions between compact objects where gravitational radiation is important. In particular we assume that the time-dependent probability density $p(M, t)$ that describes how the fraction of primordial MACHOs with a mass between $M$ and $M+d M$ changes with time is mainly due to due to gravitational radiation induced coalescence of MA$\mathrm{CHO}$ pairs, and this increase is described by a Boltzmann-like equation:

$\frac{d p(M)}{d t}=v(t) \frac{\rho_{D M}}{\bar{M}} \int_{M_{D M}}^{\infty} \pi b_{c a p}^{2} p\left(M^{\prime}\right) p\left(M-M^{\prime}\right) d M^{\prime}$

where $\rho_{D M}$ is the dark matter density at time $t, v(t)$ is the relative velocity of compact objects at time $t$ and $b_{\text {cap }}\left(M^{\prime}, M-M^{\prime}\right)$ is the critical impact parameter that would allow two compact objects to be captured into a stable orbit via gravitational radiation [11]. We believe that radiative capture is the most important mechanism for shaping the mass spectrum since the typical impact parameter is typically much larger than the Schwarzschild radius. In Eq. 1 time can run run from the time corresponding to a redshift $z_{r}$ (vis. $\sim 10^{-4} \mathrm{sec}$ ) to the present time. However we have found that the present day MACHO mass spectrum is largely fixed in the first few minutes after the big bang; i.e. by the time cosmic helium was produced. What to assume for the relative velocity $v(t)$ is of course problematic. 
However as a rough approximation we have used the "virial" $v=\left[(1+z) / 10^{12}\right]^{1 / 2} 10^{5} \mathrm{~km} / \mathrm{sec}$; i.e. we assume $v^{2}(t)$ scales roughly as the inverse of the distance between dark matter particles and reaches typical halo velocities by the time of matter dominance $z<10^{4}$. Some sample solutions of Eq. 1 assuming $p(M, t)$ at $z=z_{r}$ is concentrated at $M=M_{D M}$ is shown in Fig 1.

MACHO normalized $p(M)$

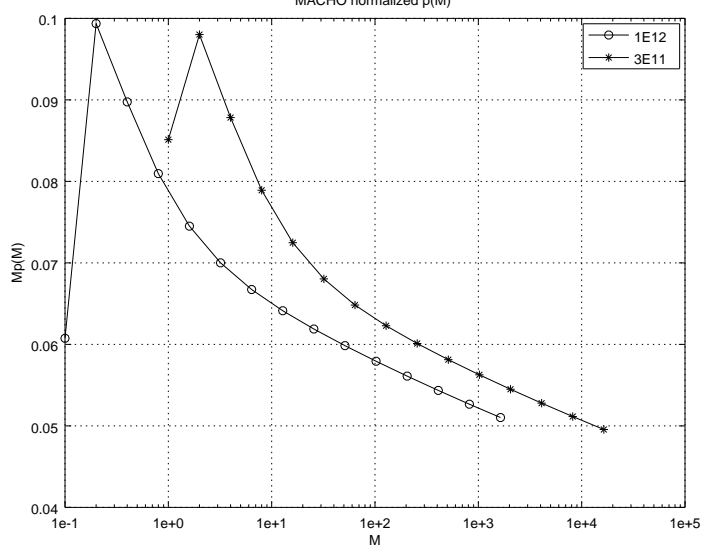

FIGURE 1: Dark matter mass spectra for $M_{D M}=.1$ and $1 M_{\odot}$ and $z_{r}=1.0 \times 10^{12}$, and $z=3.0 \times 10^{11}$.

Although we could use in Eq.1 to calculate the mass spectrum for any combination of initial mass $M_{D M}$ and redshift $z_{r}$ we have focused on the spectrum of compact objects resulting from collisions between primordial compact objects with initial masses, $M_{D M}$ in the range of $.01-1 M_{\odot}$ and values of $z_{r}>10^{10}$. Fig. 1 shows two examples of such calculations which assumed $M_{D M}=0.1$ and $1.0 M_{\odot}$. It can be seen that the spectrum is fairly flat, and that no logarithmic interval in mass accounts for more than $\sim 10 \%$ of the dark matter density. This makes our prediction consistent with a previous direct search for dark matter compact objects in the halo of our galaxy using gravitational micro-lensing [12],[13] as well as widely accepted astrophysical constraints on what typical MACHO masses might be [14],[15]. Our predicted spectrum extends to $10^{4} M_{\odot}$ with sufficient strength to possibly explain the origin of galaxies [7],[8],[9]

It is the flatness of our predicted mass spectrum that allows our predicted mass spectrum to evade previous claims cf. (see [14],[15]) that massive compact objects are excluded as candidates for dark matter. Indeed it was recently noted [? ] that a flat spectrum effectively negates the claim that a dark matter density is inconsistent with searches for micro-lensing of supernovae. The mass spectra shown in Fig. 1 also appear to evade the astrophysical constraints discussed in Ref. [14] (cf. Fig. 5) if one interprets the constraints shown in Ref. [14] for a monochromatic spectrum as constraints on the integral of our probability density over some $\log M$ interval, say $\Delta \log M=1$. On the positive side there already may be direct evidence for the existence of primordial compact objects. Partly as a result of the unexpected observation of gravitational radiation from the coalescence of two compact objects with estimated masses near to $10 M_{\odot}$, there is considerable interest at the present time in the question whether the compact objects observed by LIGO could be primordial in origin [17]. Even if the LIGO objects are primordial, the mass $M_{D M}$ is still undetermined because the LIGO objects may have formed by the coalescence of smaller compact objects. The particular choices for $M_{D M}$ shown in Fig. 1 were chosen keeping in mind that the relative abundance of dark matter objects with masses $\sim 10^{4} M_{\odot}$ should explain the observed abundance of galaxies. In particular, we assume that our predicted mass spectrum should at least conform with current estimates [7],[8],[9] that the galactic seeds should represent $\sim 10^{-4}-10^{-5}$ of the dark matter density.

Since the conventional view is that black holes are indestructible what physical meaning can be ascribed to our assumption that present day dark matter evolved from a population of PBHs at a time $\sim 10^{-4} \mathrm{sec}$ ? The answer to this question may lie with the answer to the puzzle as to the physical process that could have led to the radiation precursor to the $\mathrm{CMB}$ and how does one explain the specific entropy of the CMB. In principle inflation theories can produce radiation via reheating [10] or dissipation [18] and hybrid inflation models are capable of producing massive PBHs $[19,20]$. However to our knowledge inflation theories have to date not provided any explanations for the specific entropy of the $\mathrm{CMB}$ as measured by the number of $\mathrm{CMB}$ photons per gram of dark matter. This number is nearly the same at redshift $z_{r}$ as today, and so the mystery is really to not just explain the sudden appearance of PBHs but also explain the why these PBHs were accompanied by a specific number of photons and lepton pairs.

Our proposal for resolving this enigma is based on the observation that if $M_{D M}$ lies in the range of $\sim 0.1-1 M_{\odot}$, then the estimated radiation temperature at $z=z_{r}$ is very close to the temperature where, due to quantum effects, the surface of the compact object would no longer be transparent to the radiation as it would be in classical general relativity. Indeed it has been predicted [21] that due to quantum effects when the photon energy reaches a critical value $\approx 300 \mathrm{MeV}\left(\mathrm{M}_{\odot} / \mathrm{M}\right)^{1 / 2}$ it will strongly interact with the surface modes of the compact object. This would allow compact objects to interact strongly with radiation, and as a consequence of the very large heat capacity of such objects, would transform ambient radiation into entropy stored in the surface modes of the compact objects. As a corollary one can make an independent estimate for the critical redshift $z_{r}$ :

$\left(1+z_{r}\right) \hbar v_{C M B} \approx 0.3 \mathrm{GeV}\left(M_{\odot} / M_{D M}\right)^{1 / 2}$.

This estimate is consistent with the estimates based on MA$\mathrm{CHO}$ mass spectra that are consistent with astrophysical constraints. Thus we are led to the hypothesis that prior to the redshift $z_{r}$ the matter in the universe consisted almost exclusively of black holes rather than a mixture of PBHs and radiation.

Curiously, we extrapolate the present day energy density of the CMB $\left(.026 \mathrm{GeV} / \mathrm{cm}^{3}\right)$ to a redshift $\sim 10^{12}$, then the energy density $\rho_{r}$ is close to the energy density of an Einsteinde Sitter universe at the same epoch. In other words the total matter mass-energy density of our universe at the redshift $z_{r}$ is apparently very nearly the same as in a flat Robertson-Walker universe containing only cold matter. This means that prior to $z_{r}$ cosmological matter could have consisted entirely of black holes. Serendipitously today's observed density of dark matter is also completely consistent with the hypothesis that $\mathrm{PBH}$ pre- 
cursors to todays dark matter MACHOs arose from the decay of massive compact objects.

In the scenario for the production of massive PBHs described in Ref. [3] it was assumed that at some early epoch a matter in the universe was dominated by matter with a Frautschi-Hagedorn equation of state (which is the appropriate equation of state for a gas of black holes). Very massive compact objects are then continuously formed as a result of large density fluctuations due to Poisson fluctuations in a universe dominated by initially close packed black holes. Indeed the black hole dominated universe described in ref. [3] has the property that any sphere with radius $r_{0}$ where there is a small positive density $\delta \rho$ in mass density - no matter how small the increase in density - the sphere will not expand indefinitely, but will reach a maximum radius $R_{\max } \sim R_{0}(\rho / \delta \rho)$, where the density inside the sphere is approximately $3 \times$ the ambient mass density. Subsequently the sphere will collapse to form a black hole. All compact objects formed during the Einstein-de Sitter -like epoch will have a mass close to $M_{H}$ because any compact object mass with a smaller mass will get "swept up" into the collapse of the largest compact object formed by the collapse of the mass within the horizon $\approx 0.5(c t)$. Combining Eq. 2 with the observed energy densities for the CMB $\left(.026 \mathrm{eV} / \mathrm{cm}^{3}\right)$ and dark matter density $\left(\approx 1 \mathrm{keV} / \mathrm{cm}^{3}\right)$, yields an estimate for mass of the horizon black holes at $z=z_{r}$ :

$M_{H}\left(z_{r}\right)=20\left(\frac{1+z_{r}}{10^{12}}\right)^{4} M_{\odot}$

Although other explanations for the origin of the present day MACHOs and CMB may eventually emerge, the simplicity of the idea that both present day spectrum of MACHOs and the $\mathrm{CMB}$ are the result of the release at $z=z_{r}$ of internal energy by black holes with the mass in (3) is attractive. This scenario also provides prediction for the specific entropy of the CMB [22]. This predicted entropy was not inevitable but is a consequence of the large mass difference between the horizon mass in Eq. 3 and the residual mass $M_{D M}$ estimated from the current constraints on the MACHO mass spectrum. This scenario relies on the very large heat capacity of compact objects, and provides a natural explanation for the somewhat mysterious circumstance that at the present time matter consists of a component with large entropy (the $\mathrm{CMB}$ ) and a dark matter component with seemingly low entropy (discounting the unobservable Hawking entropy). We also note that as a result of the sweeping up of all matter into massive black holes prior to $z=z_{r}$ no primordial WIMPs, SUSY monopoles, or other exotic elementary particles will survive into the observable era $z<z_{r}$.

During the epoch $z>z_{r}$ we hypothesize that cosmological matter will consist mostly of massive black holes. Since the cosmological pressure due to black holes is negligible the mass density, the mass-energy density for $z>z_{r}$ will vary in the familiar way when mass is conserved $\rho=\rho_{r}\left[(1+z) /\left(1+z_{r}\right)\right]^{3}$. Combining this with the relationship between time and redshift for a Roberston-Walker universe with zero pressure $t / t_{r} \approx$ $\left[\left(1+z_{r}\right) /(1+z)\right]^{3 / 2}$ provides an estimate for the horizon scale masses prior to $z=z_{r}$ :

$M_{H}=\left(\frac{1+z_{r}}{1+z}\right)^{3 / 2} M_{H}\left(z_{r}\right)$.

We see that the horizon mass decreases as $\mathrm{z}$ increases, and so the mass-energy density would diverge in the limit $z \rightarrow \infty$ if only horizon scale black holes were present. However if we assume that the horizon scale black holes were the result of the coalescence of smaller black holes, then the mass-energy density will have a finite maximum $\rho_{*}$ which is attained when the smaller black holes become close packed. In a sense all the large scale features of our present day universe are determined by the value of $\rho_{*}$. It is perhaps worth mentioning the similarity of this scenario to Zeldovich's cosmological model [23], where he assumed that the universe began with close packed nucleons. On the other hand, our mechanism for the production of the $\mathrm{CMB}$ is rather different from Zeldovich's.

As a result of renewed efforts to use gravitational microlensing to detect compact objects with masses $>M_{\odot}$ it may not be too far off before we know what the present day MA$\mathrm{CHO}$ spectrum looks like. Combining this knowledge with solutions to Eq. 1 one can determine the total mass-energy density $\rho_{r}$ at redshift $z_{r}$. Unfortunately the initial energy density $\rho_{*}$ corresponding to the time when the initial PBHs for the $z>z_{r}$ era become close packed is undetermined. However the distinct possibility that the initial PBHs may have originated at an finite red-shift $z_{*} \gg z_{r}$ suggests that the emergence of our expanding universe is associated with a phase transition of the vacuum state at some energy density $\gg(\mathrm{GeV})^{4}$. It is tempting to speculate at this initial energy density may be related to the breaking of supersymmetry. Finally we note that our scenario does not preclude a inflationary episode preceding $z_{*}$. However from our point view that our observed universe began with a universe filled with black holes, it is perhaps more natural to note [24] that the "flatness" problem can be solved by assuming the the era $z_{*}>z>z_{r}$ was preceded by an era where the universe was flat and contained matter, but the vacuum energy was negative.

\section{Acknowledgements}

The authors are very grateful for discussions with Carlos Frenk, Paul Frampton, Michael Schneider, Will Dawson, and Nathan Golovich. This work was performed under the auspices of the U.S. Department of Energy by Lawrence Livermore National Laboratory under Contract DE-AC52-07NA27344 and was supported by the LLNL-LDRD Program under Project No. 17-ERD-120.

\section{References}

[1] S. Hawking, Nature, 248, 30, (1974)

[2] G. Chapline, Nature, 253, 251, (1975)

[3] G. Chapline, Phys. Rev., D12, 2949, (1975)

[4] P. H. Frampton, Mod. Phys. Lett., bf A31, 1650093, (2016)

[5] G. Chapline and P. H. Frampton, JCAP, 11, 916, (2016)

[6] K. Greist, Ap. J., 366, 412, (1991)

[7] J.E. Green, Nature Communication, DOI:10.1038 /ncomms 2314

[8] J. Schaye et. al. Mon. Not. Roy. Soc., 446 521, (2015)

[9] B. Carr and J. Silk, "Primordial black holes as seeds for cosmic structures", arXiv:1802.006v1[astro-ph], (2018)

[10] E. W. Kolb and M. S. Turner The Early Universe, (AddisonWesley 1990)

[11] Y-B, Bae, et.al."Gravitational driven capture in unequal black hole encounters", arXiv:1701.01548v2[astroph], (2017) 
[12] C. Alcock, et. al., Ap.J, 499, 19, (1998)

[13] P. Tisserand et .al., Astron., Astrophys., 469, 387, (2007)

[14] B. Carr, F. Kuhnel, and M. Sandstad, Phys. Rev , D94 08350, (2016)

[15] B. Carr, et. al., Phys Rev., D96, 023514, (2017)

[16] S. Clesse and J. Garcia-Bellido, arXiv:1712.06574 [astro-ph] , (2017)

[17] S. Bird, et.al., Phys Rev Lett., 116, 20011301, (2016)

[18] A. Berera, Nucl. Phys. B, 585, 666, (2000)
[19] P. Framption e. al., JCAP,1004 023, (2010)

[20] S. Clesse and J. Garcia-Bellido, Phys.. Rev., D92, 023525, (2105)

[21] G. Chapline, E. Hohlfeld, R. Laughlin, and D. Santiago, Phil. Mag., B81, 235, (2001)

[22] G. Chapline, OAlib J., 1101174, (2010)

[23] Y. B. Zeldovich, Mon. Not. Roy. Astro. Soc., 160, 17, (1972)

[24] G. Chapline and J. Barbieri, "Was there a negative vacuum energy in your past", arXiv:1602.01152[gr-qc], (2016) 